\title{
On Predicting Form and Meaning in a Second Language
}

\author{
Aine Ito, Andrea E. Martin, and Mante S. Nieuwland \\ University of Edinburgh
}

\begin{abstract}
We used event-related potentials (ERP) to investigate whether Spanish-English bilinguals preactivate form and meaning of predictable words. Participants read high-cloze sentence contexts (e.g., "The student is going to the library to borrow a ..."), followed by the predictable word (book), a word that was form-related (hook) or semantically related (page) to the predictable word, or an unrelated word (sofa). Word stimulus onset synchrony (SOA) was $500 \mathrm{~ms}$ (Experiment 1) or $700 \mathrm{~ms}$ (Experiment 2). In both experiments, all nonpredictable words elicited classic N400 effects. Form-related and unrelated words elicited similar N400 effects. Semantically related words elicited smaller N400s than unrelated words, which however, did not depend on cloze value of the predictable word. Thus, we found no N400 evidence for preactivation of form or meaning at either SOA, unlike native-speaker results (Ito, Corley et al., 2016). However, non-native speakers did show the post-N400 posterior positivity (LPC effect) for form-related words like native speakers, but only at the slower SOA. This LPC effect increased gradually with cloze value of the predictable word. We do not interpret this effect as necessarily demonstrating prediction, but rather as evincing combined effects of top-down activation (contextual meaning) and bottom-up activation (form similarity) that result in activation of unseen words that fit the context well, thereby leading to an interpretation conflict reflected in the LPC. Although there was no evidence that non-native speakers preactivate form or meaning, non-native speakers nonetheless appear to use bottom-up and top-down information to constrain incremental interpretation much like native speakers do.
\end{abstract}

Keywords: non-native language comprehension, prediction, N400, LPC, SOA

What does it mean to understand a language? At the very least, it means to know the meaning of its words and to know which sentences are allowed by its grammatical rules. Yet those things alone are clearly not enough to engage in a regular conversation. Beyond achieving intricate and often nonliteral meanings, a listener needs to access words and apply rules to establish the intended meaning in a timely manner, in order to keep up with the pace of the speaker. That is, the listener needs to process language incrementally, by using relevant information as soon as possible, and sometimes perhaps even predictively, by anticipating information where possible. Native speakers of a language typically do all of this without any conscious effort. However, it does not follow that incremental and predictive processing is necessary for successful comprehension (for discussion, see Huettig \& Mani, 2015). For example, non-native speakers may show slower and/or less predictive processing compared with native speakers, though ultimately understanding the sentence correctly. We therefore tested whether fluent non-native speakers exhibit incrementality or to what degree they preactivate information during sentence processing.

This article was published Online First September 26, 2016.

Aine Ito, Andrea E. Martin, and Mante S. Nieuwland, Department of Psychology, School of Philosophy, Psychology and Language Sciences, University of Edinburgh.

Correspondence concerning this article should be addressed to Aine Ito, Department of Psychology, School of Philosophy, Psychology and Language Sciences, University of Edinburgh, 7 George Square, Edinburgh EH8 9JZ, Scotland, United Kingdom. E-mail: aine.ito@ed.ac.uk
The current study used the same approach and experimental design as Ito, Corley, Pickering, Martin, and Nieuwland (2016). Ito, Corley et al. (2016) conducted two event-related potential (ERP) studies on written sentence comprehension to examine whether native speakers anticipate the form and meaning of upcoming words. Their participants read constraining sentence contexts that were followed by either a predictable word or an implausible word. The implausible word was related to the predictable word in either word form or meaning, or was unrelated to the predictable word. Although all types of implausible word elicited an N400 effect relative to the predictable word (e.g., Kutas \& Hillyard, 1980), the N400 effect was smaller for semantically related words than for unrelated words. When participants read sentences at $700 \mathrm{~ms}$ word stimulus onset asynchrony (SOA), but not at 500-ms SOA, the N400 effect for form-related words was also smaller than that seen for unrelated words. Critically, these N400 reductions depended on the cloze probability of the predictable word, whereas they did not depend on the plausibility of the encountered word. Based on this pattern, the N400 reductions were taken as evidence for prediction of form and meaning, rather than merely reflecting easeof-integration (see Federmeier \& Kutas, 1999, for a similar argument). In a prediction-account, the facilitation of semantic processes as reflected in the N400 is driven by the strength of prediction and the extent to which encountered input matches this prediction. In an integration-account, facilitation of semantic processes ought to correlate with the sentence plausibility.

Ito, Corley et al. (2016) also found a post-N400 positivity (late positive component effect) for form-related words relative to all other types of words, which also depended on cloze probability of the predictable word. The effect was interpreted as showing detection of form conflict between the predictable and form-related 
words. This effect can occur in the absence of prediction (i.e., independently of N400 effect reductions), because the predictable word can become activated after the form-related word is retrieved, and reflects a top-down effect of contextual meaning on integrative processing, or a sort of "analysis by synthesis" (Martin, Monahan, \& Samuel, 2016; Townsend \& Bever, 2001). Using the Ito et al. design, the current study thus examined preactivation of form and meaning in non-native speakers.

\section{Incremental Processing During Non-Native Language Comprehension}

There is mixed evidence as to whether non-native speakers comprehend sentences incrementally like native speakers. Several visual world eye-tracking studies have found evidence for incrementality in non-native language comprehension. For example, Trenkic, Mirković, and Altmann (2014) found that late Mandari$\mathrm{n}$-English bilinguals used information conveyed by English articles to constrain referential domains and resolve reference (although they were overall slower than native speakers). In contrast, Lew-Williams and Fernald (2010) found that, whereas native Spanish speakers rapidly used the grammatical gender of articles preceding a noun to identify an upcoming referent, late English-Spanish bilinguals did not take advantage of grammatical gender information. Evidence from reading studies is also not clear-cut. There is evidence for incremental processing in nonnative speakers (Jackson \& Bobb, 2009; Roberts \& Felser, 2011), but native-like incrementality in non-native speakers has been shown to depend on their proficiency (Hopp, 2006), working memory span (Dussias \& Pinar, 2010), or experimental task (Williams, 2006).

ERP studies also show mixed results. Non-native speakers often show qualitatively similar N400 effects to native speakers in response to semantic anomaly (Kotz, 2009). Native-like N400 effects have also been reported for plausible, relatively unexpected words relative to highly expected words (Foucart, Martin, Moreno, \& Costa, 2014; Martin et al., 2013), suggesting that non-native speakers can comprehend meaning incrementally like native speakers. However, the observed N400 effects are sometimes delayed or smaller in non-native speakers relative to effects in native speakers (Martin et al., 2013; Weber-Fox \& Neville, 1996), suggestive of slower integration of word meaning in non-native speakers.

In sum, findings from multiple methodologies suggest that differences between native- and non-native speakers can manifest themselves in the time-course or size of observed effects. However, these differences do not occur consistently in the literature on non-native language comprehension.

\section{Predictive Processing in Non-Native Speakers}

Incremental comprehension is a prerequisite for generating predictions about upcoming words. If non-native speakers do not show the same degree of incrementality as native speakers, they are also less likely to predict upcoming information. In fact, though evidence for prediction in native speakers has been obtained in various studies (Huettig, 2015), evidence for prediction in non-native speakers is sparse and mixed. Some visual world eyetracking studies report predictive looks to upcoming referents in non-native and native speakers alike (Chambers \& Cooke, 2009; Hopp, 2013), whereas some studies did not observe predictive looks in non-native speakers but only in native speakers (Mitsugi \& MacWhinney, 2016). As we will discuss in more detail below, inconsistent results may be due to the proficiency of non-native speakers or to differences in the experimental manipulations.

Two recent ERP studies also reported inconsistent findings. Martin et al. (2013) found evidence for prediction in native speakers but not in non-native speakers. ${ }^{1}$ They made use of English articles a/an, and manipulated them in the way that predictable nouns (e.g., a chair following "He was very tired so he sat on ...") and plausible but unpredictable nouns (e.g., an armchair) always matched a different article. Native speakers but not non-native speakers showed an N400 effect for articles that mismatched predictable nouns relative to prediction-matched articles, suggesting that non-native speakers did not predict specific word form like native speakers. Both native and non-native speakers showed an N400 effect for unpredictable nouns relative to predictable nouns. This effect suggests that integration of predictable words was facilitated due to predictablity or plausibility, which was established from an ongoing context. This therefore supports incremental processing. However, Foucart et al. (2014) found that French-Spanish late bilinguals and Spanish native speakers can predict specific words to a similar extent. Both groups of participants elicited the same N400 effect for gender-marked articles that mismatched predictable nouns relative to prediction-matching articles (e.g., la [feminine] vs. el [masculine] when readers could expect el tesoro).

Several factors could account for the inconsistent findings in the literature, including differences in participants and experimental manipulations. Another explanation would be that non-native speakers can engage in predictive processing, but perhaps do not do so as routinely or automatically as native speakers so. One possible reason why this might be the case is that that non-native speakers must overcome several obstacles in their efforts to understand language with the richness and depth that a native speaker naturally achieves. First, for example, non-native speakers may unintentionally coactivate words from their native language (Chambers \& Cooke, 2009; Shook \& Marian, 2013). This can lead to delays in accessing words in their non-native language when coactivated words differ in meaning from the intended word (Kroll, Gullifer, \& Rossi, 2013). Second, non-native speakers appear to generate less robust or less detailed syntactic structures during comprehension (e.g., Clahsen \& Felser, 2006), and rely more on lexical-semantic relationships instead. Under this shallow structure account of Clahsen and Felser (2006), non-native comprehension is particularly impaired in sentences with relatively complex or infrequent syntactic information (e.g., resolving longdistance dependencies in English; Marinis, Roberts, Felser, \& Clahsen, 2005).

Beyond these two specific obstacles, however, a more general reason that non-native comprehension may be slower or less accurate than native comprehension is that non-native speakers are

\footnotetext{
${ }^{1}$ We recently tried to replicate these results of Martin et al. (2013) but failed to obtain any evidence for prediction in native and in non-native speakers both at a 500-ms SOA and a 700-ms SOA (Ito, Corley et al., 2016). We return to this issue in the General Discussion, where we discuss the robustness of prediction effects.
} 
typically less proficient and/or less experienced than native speakers (Kroll \& De Groot, 2005). Kaan (2014) recently argued that there are no qualitative differences in incremental and predictive comprehension between native and non-native speakers, only quantitative differences. Moreover, she suggested that those quantitative differences result from the same factors that underlie individual differences in native speaker proficiency, such as quality of lexical representations, the ability to entertain low-frequent word meanings or competing structural parses, as well as taskinduced factors. Quality of lexical representations may be particularly important as it could lead to slower lexical access and weaker semantic networks (Ivanova \& Costa, 2008). Hence, proficiency may be more important for incremental processing (Kotz \& Elston-Güttler, 2004), and therefore for predictive processing, than nativeness per se. However, proficiency levels may vary stronger in non-native speakers than in native speakers (e.g., Blumenfeld \& Marian, 2007), so that effects of predictive processing appear sporadically in reported studies. In any case, proficiency and nativeness are coextended, if not confounded.

If the mixed results on non-native predictive processing stem from proficiency differences, then studies with highly proficient participants would show effects of incremental or predictive processing (e.g., Hopp, 2013) and studies with less proficient participants would show no such effects (e.g., Mitsugi \& MacWhinney, 2016). However, other factors may matter too. Evidence for prediction may be obtained more readily if the linguistic features of the critical manipulation (i.e., the a/an distinction or grammatical gender) are also present in the native language (e.g., Foucart et al., 2014). Yet, this role of language-similarity may not be independent of language proficiency, and on its own cannot explain that prediction effects are sometimes observed under the manipulation of grammatical features that are not shared between native and non-native language (e.g., Hopp, 2013).

Details of the experimental paradigm are also relevant, and between-experiment comparisons are usually confounded by several factors. For example, it may be the case that prediction effects are more reliably elicited by some linguistic manipulations than others. If the status of a linguistic feature makes it function as a stronger cue to meaning or a higher level of representation, it might have more influence on processing than a feature that only sometimes is a cue or that does not lead to higher level of representation (Bates \& MacWhinney, 1989). For example, in Spanish, the grammatical gender of a noun is not only an important feature or cue during native acquisition and second-language learning of nouns, but it is also diagnostic during the establishment of adjacent and nonadjacent syntactic and referential dependencies in routine production and comprehension (Martin et al., 2016). In contrast, the alan distinction in English is a phonotactic rule that does not signal any lexical feature, nor cue a nonlocal dependency, rather it signals what the upcoming syllable or phoneme across the boundary between determiner and the next word will be. Furthermore, the rule can be violated during natural speech, because of its disfluency or pauses, without eliciting judgments of ungrammaticality in the same way as a grammatical gender violation would (Pullum \& Zwicky, 1988). Thus, variation in the information status of the linguistic cues that are manipulated may also contribute to the mixed pattern of results across the literature (for further discussion, see Ito, Corley et al., 2016).
In addition to representational differences, the time constraints placed upon participants during processing may also shape the observed effects. For example, the visual world eye-tracking studies that have reported evidence for prediction in non-native speakers allowed more time to generate predictions (by inserting an adjective between prediction-cueing articles and predictable nouns; Hopp, 2013) compared with studies that did not observe prediction effects (Lew-Williams \& Fernald, 2010). This is an important factor, because people are more likely to predict upcoming information when they have more time (e.g., Ito, Corley et al., 2016). Another important factor, in ERP studies particularly, is critical word predictability. The only ERP study that reported prediction effects in non-native speakers (Foucart et al., 2014) had critical nouns that were more predictable $(M$ cloze $=81 \%)$ than those used in the one study that did not find prediction effects (Martin et al., 2013; $M$ cloze $=65 \%$ ).

In sum, the mixed evidence for predictive processing in nonnative speakers may be due to multiple factors, in particular language proficiency, experimental manipulation, the status of the linguistic cues being manipulated, time constraints, and sentence predictability. Therefore, we focus not on whether or not nonnative speakers predict upcoming information, but rather on the circumstances in which they do or do not exhibit signals consistent with prediction. In the current study, we employed a design that does not principally rely on a single type of grammatical information or linguistic cue, instead we asked whether non-native speakers predict the form and meaning of upcoming words in constraining contexts and whether any effects we might find would depend on time constraints.

\section{The Present Study}

The present study adopted the experimental design used by Ito et al. (Ito, Corley et al., 2016). In that study, native speakers read highly constraining sentence contexts (e.g., "The student is going to the library to borrow a ..."), followed by the predictable word (book), an implausible word that was form-related (hook) or semantically related (page) to the predictable word, or an unrelated implausible word (sofa). Participants read sentences at 500-ms SOA, which is often used in ERP studies involving native speakers, or 700-ms SOA, which is commonly used in studies involving non-native speakers (e.g., Foucart et al., 2014; Martin et al., 2013). All types of implausible words elicited a classic N400 effect. Semantically related words elicited a smaller N400 than unrelated words at both SOAs. Form-related words also elicited smaller N400s than unrelated words, but for sentences with a very high-cloze probability and at 700-ms SOA only. Form-related words additionally elicited a post-N400 posterior positivity (LPC effect) at both SOAs. Both the observed N400 reduction and the LPC effect did not depend on the plausibility of the anomalous word itself but were dependent on the cloze probability of the predictable word.

The observed N400 results suggest that native speakers preactivated semantic and form information, but only preactivated form when they were reading relatively slowly and had very strong predictions for the upcoming word. This result suggests that preactivation of word form is less likely to occur compared to preactivation of meaning. In addition, our results suggest that, whether form was preactivated or not, participants activated the predictable word after accessing the meaning of the form-related word, leading 
to a conflict in interpretation reflected in the observed post-N400 LPC effect (Nieuwland, 2015; Van de Meerendonk, Kolk, Chwilla, \& Vissers, 2009). Following Ito, Corley et al. (2016), we do not regard this LPC effect as evidence for preactivation of form information, because people can detect such a conflict without predicting word forms. Instead, this pattern arises from a combination of top-down activation of contextual meaning and bottom-up activation of word form information due to formsimilarity. For example, the context "The student is going to the library to borrow a ..." can activate meaning of the word book more strongly compared with other words that are unrelated to the context. Reading a form-related word hook will spread activation (via priming) to the word form of the predictable word book, which fits highly plausibly (and much more plausibly compared to hook) into the given context. This will then trigger a conflict between the encountered word and the (unseen) highly predictable and plausible word.

Our current predictions are derived from the results from native speakers. If non-native speakers preactivate form and meaning of upcoming words, we expect to see reduced N400s to form-related and meaning-related critical words compared to unrelated words. Such effects would need to be independent of plausibility but dependent on the cloze value of the predictable word to constitute evidence for preactivation. If N400 reduction is dependent on both plausibility and cloze value, it may reflect facilitation due to higher plausibility and/or due to similarity with predicted information. Thus, the effect would be compatible with both integration and prediction accounts. Like in native speakers, we hypothesized that preactivation of form in non-native speakers is less likely to occur and more dependent on reading rate than preactivation of meaning. This hypothesis is based on the assumption that preactivation of form would need to be mediated by prediction of a specific word (because contexts do not normally have relationship in word form at more than chance level), while preactivation of meaning needs not (because contexts can preactivate relevant meaning without causing prediction of a specific word).

Relevant to this hypothesis is the fact that ERP studies on written language comprehension in non-native speakers typically use a slower SOA than studies on native comprehension, because the standard 500-ms SOA can be an uncomfortably fast pace for non-native speakers. We therefore expected to find no evidence of preactivation in our first experiment that used a 500-ms SOA. However, participants may nevertheless activate the predictable word after encountering the form-related word, as would be evidenced by an LPC effect to the form-related condition.

\section{Experiment 1}

\section{Method}

Participants. Twenty-four Spanish-English bilinguals (eight men and 16 women; age: $M=26.3$ years, $S D=4.6$ ) participated in the experiment. All participants were tested at University of Edinburgh, and all were native speakers of Spanish. One participant was excluded from analysis due to a large portion of data artifacts. All participants were right-handed and reported no neurological or language disorders. The mean age of their first exposure to English was 11.0 years $(S D=7.6)$, and the mean length of their exposure to English was 12.5 years $(S D=5.9)$. Their overall self-rated English proficiency score was $8.2(S D=0.7$; rated on a scale from 1 to 10$).^{2}$

Stimuli and experimental design. The stimuli and experimental design were identical to those used in Ito, Corley et al. (2016). Example sentences are shown in Figure 1, and a full list of the experimental sentences are in the Appendix. The experimental sentences were 160 items that consisted of a context that predicted a specific word (based on a cloze test in native speakers), followed by one of four types of critical word, and a sentence final word. In the predictable condition, the critical word was the predictable word. In the form condition, the critical word was related to the predictable word in phonological and orthographic form. The form overlap with the predictable word occurred at word onset (card-cart, 15\% of the items), at word offset (luck-duck, 53.8\%), or both (age-ace, $23.8 \%$ ), or involved single-letter addition (air-hair, 5.6\%) or singleletter deletion (cold-old, $1.9 \%$ ). In the semantic condition, the critical word was semantically related to the predictable word (we assessed semantic relatedness using LSA values, taken from http://lsa.colorado .edu/). In the unrelated condition, the critical word was not related to the predictable word in form or meaning.

We collected new cloze probability values from a group of similar pool of participants as for the ERP experiments (12 Spanish-English bilinguals resident in Edinburgh, who did not take part in the ERP experiment). Participants completed the experimental sentences truncated before the critical word (e.g., The student is going to the library to borrow a ...), using the first word that came to mind. The mean non-native cloze value was $61 \%$ (range $=0 \%-100 \% ; S D=25.4)$, which was lower than the mean native cloze value of $80 \%$ (range $=31 \%-100 \%$ ).

We collected new plausibility values from another group of similar pool of participants as for the ERP experiments (a total of 20 Spanish-English bilinguals who did not take part in the ERP experiment or in the cloze pretests), following the procedure described in Ito et al. The mean plausibility ratings for each condition are displayed in Figure 1. The semantic condition was rated more plausible than the form condition (difference: $M=.26$, $S D=.56), t(159)=3.7, p<.001$, or the unrelated condition, (difference: $M=.36, S D=.83), t(159)=5.5, p<.001$, which was consistent with the native speaker ratings as in Ito et al. The form condition was rated marginally more plausible than the unrelated condition (difference: $M=.10, S D=.68$ ), $t(159)=1.9$, $p=.06$, whereas the form condition and the unrelated condition did not differ in the native speaker ratings. ${ }^{3}$

Procedure. The 160 sentences were divided into four counterbalanced lists so that each list contained only one condition per sentence, but that across the four lists each condition for each sentence occurred equally often. They were combined with 64

\footnotetext{
${ }^{2}$ There appeared to be a large variation in non-native speakers' proficiency. To check whether the effects of interest are affected by a small number of participants whose proficiency was not high, we conducted additional analyses after excluding participants whose length of exposure to English was 4 years or shorter.

${ }^{3}$ The slightly higher plausibility ratings for the form condition were mainly driven by 10 items, in which form-related words had plausibility of three or above. In the remaining 150 items, the plausibility did not differ between the form $(M=1.59)$ and the unrelated condition $(M=1.58)$, $t(149)=.26, p>.1$.
} 
Non-native speakers: All items (500 ms SOA)
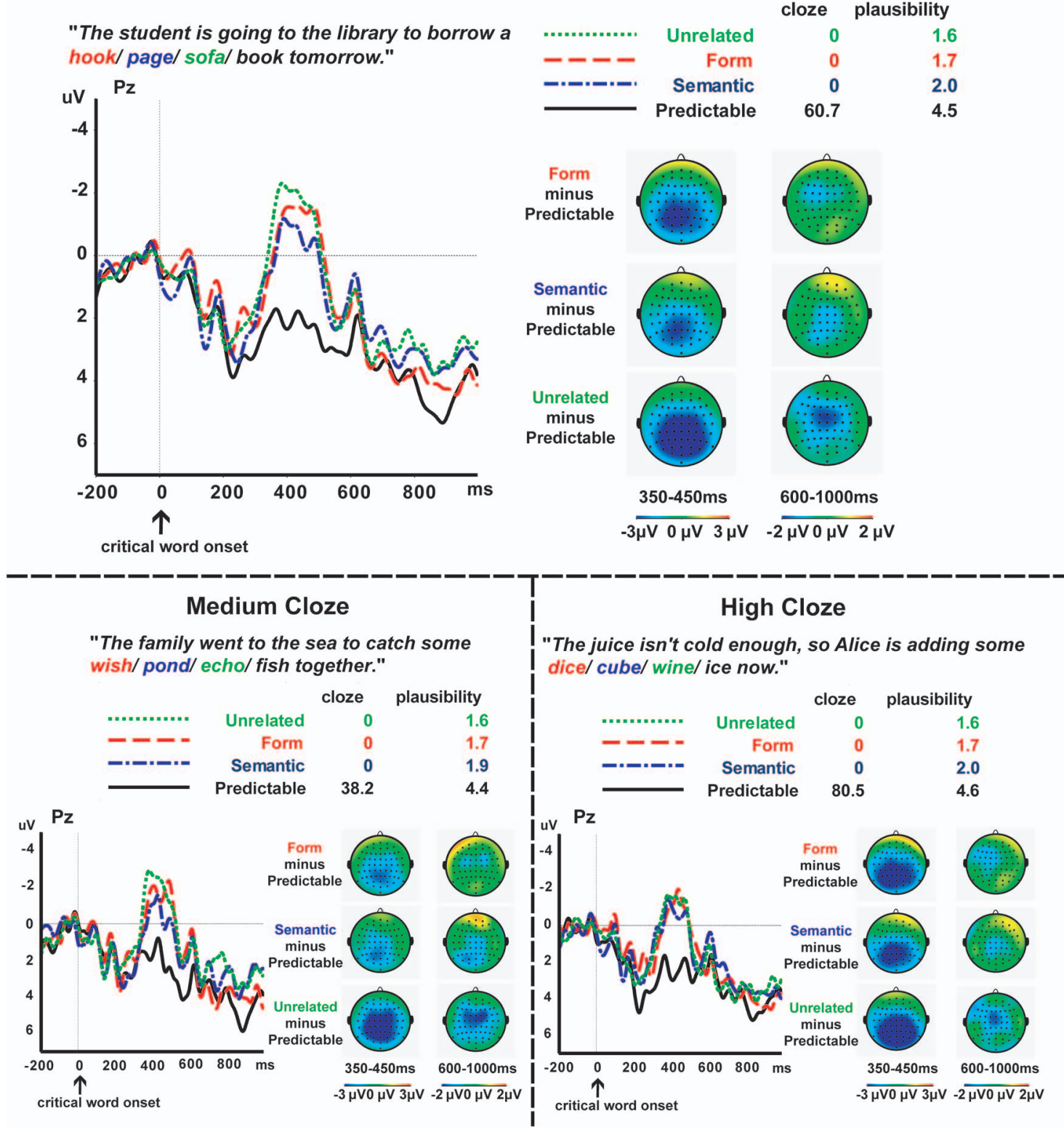

Figure 1. Results from Experiment 1 (500-ms stimulus onset asynchrony [SOA]). Event-related potentials (ERPs) elicited by each condition at $\mathrm{Pz}$ in all items (top panel), in medium-cloze items (left lower panel), and in high-cloze items (right lower panel). Scalp distributions of the ERP effects (implausible condition minus predictable condition) in the N400 time window and LPC time window are shown on the right in each panel. See the online article for the color version of this figure.

additional plausible filler sentences and presented in the same randomized order for every participant with the constraint that no more than three items from the same condition appeared consecutively. Each participant thus saw a total of 104 plausible and 120 implausible sentences.

Participants silently read sentences from a computer display, presented word by word at a regular pace (300-ms word duration, 200-ms interword interval; sentence final words had a 600-ms duration). A fixation-cross followed each sentence, at which point participants could start the next sentence by a button-press. Yes-No comprehension questions appeared on $25 \%$ of the trials. These questions were not about critical words.

Mean accuracy for comprehension questions during the ERP experiment was $93.6 \%(S D=6.4 ; 8.6 \%$ of the data was excluded because of time-outs). The mean number of artifact-free trials in Experiment 1 per condition was $37(S D=.24)$, with no difference across conditions. Before the EEG session, participants completed a language background questionnaire. 
Data processing and analysis. The electroencephalogram (EEG) was recorded at a sample rate of $512 \mathrm{~Hz}$ and with 24-bit AD conversion using the Biosemi ActiveTwo system (BioSemi BV, Amsterdam, The Netherlands). This system's hardware is completely DC coupled and applies digital low pass filtering through its analog-to-digital converter (ADC) decimation filter (the hardware bandwidth limit), which has a fifth-order sinc response with $\mathrm{a}-3-\mathrm{dB}$ point at one fifth of the sample rate (i.e., approximating a low-pass filter at $100 \mathrm{~Hz}$ ). Data were recorded from 64 EEG, 4 EOG, and two mastoid electrodes using the standard 10/20 system (for details, see Nieuwland, 2014). Offline, the EEG was re-referenced to the mastoid average and filtered further $(0.019-20 \mathrm{~Hz}$ plus $50 \mathrm{~Hz}$ Notch filter). Data was segmented into $1,200-\mathrm{ms}$ epochs ( -200 to $1,000 \mathrm{~ms}$ relative to critical word onset), corrected for eye-movements using the Gratton and Coles regression procedure as implemented in BrainVision Analyzer (Brain Products ${ }^{\odot}$ ), baseline-corrected to -100 to $0 \mathrm{~ms}$, automatically screened for movement- or electrode-artifacts (minimal/maximal allowed amplitude $=-75 / 75$ $\mu \mathrm{V}$ ), and averaged per condition per participant.

As in Ito, Corley et al. (2016), mean amplitude was computed per condition at 16 EEG electrodes (F1/F3/FC1/FC3/CP1/CP3/ $\mathrm{P} 1 / \mathrm{P} 3$ plus right-hemisphere equivalents), in the N400 time window (350-450 ms) and the LPC time window (600-1,000 ms). Effects of condition and scalp distributions effects were tested with a 4 (condition: predictable, form, semantic, unrelated) $\times 2$ (hemisphere: left, right) $\times 2$ (anteriority: frontal-central, central-parietal) repeated-measures ANOVA. When appropriate, Greenhouse-Geisser corrections and corrected $F$ values are reported. Only statistical results with $p<.1$ are reported. We additional performed analyses, by dividing the items into high- and medium-cloze probability sets, by removing very low-cloze items, and by removing low-proficiency participants. Details of these analyses are described in the section for each analysis.

\section{Results}

Visual inspection of Figure 1 indicated that all implausible conditions elicited larger N400s than the predictable condition. These N400 effects had a broad central-posterior distribution. Unlike the findings in native speakers, we did not observe an LPC effect for form-related words.

The N400 time window. N400 analysis revealed a significant effect of condition, $F(3,66)=12.0, M S E=12.1, p<.001$, and a significant interaction of condition by anteriority, $F(3,66)=6.8$, $M S E=1.5, p<.001$, indicating that effects of condition were more robust at posterior channels, $F(3,66)=16.8, M S E=3.4$, $p<.001$, than at anterior channels, $F(3,66)=6.2, M S E=3.5$, $p<.001$. Given the stronger effects at posterior channels, we proceeded with pairwise $t$ tests between conditions focused on posterior channels. All the anomalous conditions elicited larger $\mathrm{N} 400$ s than the predictable condition $(p \mathrm{~s}<.001)$. The semantic condition elicited smaller N400s than the unrelated condition, $(M=-1.1 \mu \mathrm{V}, S D=2.7), t(45)=-2.8, p<.01$, and the form condition elicited marginally smaller N400s than the unrelated condition, $(M=-.75 \mu \mathrm{V}, S D=2.7), t(45)=-1.9, p=.07$. There was no difference between the form and semantic conditions $(p>.1)$.
The post-N400 time window. The post-N400 window analysis revealed a significant condition by anteriority interaction, $F(3$, $66)=4.0, M S E=1.7, p=.01$, and a marginally significant condition by hemisphere, $F(3,66)=2.3, M S E=.28, p=.08$. We followed up with one-way ANOVAs conducted at anterior and posterior channels separately, which revealed that the effect of condition was marginally significant at anterior channels only, $F(3,66)=2.7, M S E=3.7, p=.05$.

Effects of cloze probability. We compared effects of condition on N400 and LPC in high-cloze and medium-cloze items, following the same procedure used in Ito, Corley et al. (2016) Using the median-cloze probability collected from the non-native speaker group $(M d n=66)$, the items were split into a high-cloze subset ( 85 items; cloze $M=80.5, S D=10.3$ ) and a medium-cloze subset (75 items; cloze $M=38.2, S D=17.6$ ). Plausibility ratings in the predictable condition were higher in the high-cloze than for the medium-cloze items, $t(146)=2.4, p<.05$, but plausibility in the anomalous conditions did not differ $(p s>1)$. Frequency, word length, Levenshtein distance, word LSA or context LSA did not differ between the high-cloze and the medium-cloze subsets $(p s>.05)$.

In this analysis, we followed Ito, Corley et al. (2016) and included posterior channels only, where N400 and LPC effects were found to be the most robust. For the N400 amplitudes per condition, a two-way condition by cloze ANOVA revealed a significant effect of condition, $F(3,66)=16.7, M S E=6.6, p<$ .001 , and a marginally significant effect of cloze, $F(1,22)=3.0$, $M S E=6.5, p=.1$, but no Condition $\times$ Cloze interaction $(F<1)$. For the LPC time window, a condition by cloze ANOVA revealed no significant effect or interaction involving cloze $\left(F_{\mathrm{S}}<2.4\right)$.

The non-native cloze values were very low for some of the experimental sentences (range $=0 \%-100 \%$ ), in contrast to the native cloze values in Ito, Corley et al. (2016) (31\%-100\%). To test whether the current lack of N400 reduction/LPC effects arose from including these low-cloze items, we reanalyzed our data by excluding items with cloze values lower than $30 \%$. This left 138 items (cloze $M=68 \%$ ), and the mean cloze values for the new high- and medium-cloze items were $84.7 \%(N=65)$ and $53.1 \%$ $(N=73)$, respectively. We conducted the same ANOVA on the new item sets, testing the interaction of condition by cloze in the $\mathrm{N} 400$ and the post-N400 time windows. The analysis in the N400 time window revealed no main effect of cloze $(F<1)$, or interaction of condition by cloze $(F<1.5)$. The analysis in the post-N400 time window revealed no significant main effects or interaction $\left(F_{\mathrm{S}}<1.3\right)$. Thus, the patterns of the results remained the same even when low-cloze items were removed.

We also considered a possibility that a few participants who had relatively low proficiency contributed to the lack of N400 reduction/LPC effects, and conducted the same ANOVA on the subset of participants whose length of exposure to English was longer than 4 years (19 participants). Neither the N400 time window nor the post-N400 time window showed any effect or interaction involving cloze $(F \mathrm{~s}<1)$.

\section{Discussion}

In Experiment 1, implausible words that were semantically related to predictable words elicited reduced N400s compared with unrelated control words. Implausible words that were form-related to 
predictable words also elicited slightly reduced N400s compared with unrelated words. However, none of the observed effects depended on cloze probability of the predictable word. We do not take these effects as evidence for preactivation because they could reasonably be explained in terms of plausibility (e.g., the semantically related condition was more plausible than the unrelated condition). The results of Experiment 1 thus suggest that participants did not preactivate semantic or form information of upcoming words.

\section{Experiment 2}

We considered the possibility that the 500-ms SOA in Experiment 1 was too fast for non-native speakers to read comfortably. Wlotko and Federmeier (2015) found that native speakers showed N400 reduction for words that were semantically related to predictable words at 500-ms SOA, but this effect was diminished at 250-ms SOA (see also Dambacher et al., 2012). These studies suggest that uncomfortably fast SOA may burden the comprehension system in general, resulting in smaller prediction effects. It is well-established that nonnative speakers, even when highly proficient, read more slowly than native speakers (Hopp, 2009). Probably for this reason, ERP studies on non-native sentence comprehension typically use an SOA that is slower than the standard 500-ms SOA used with native speakers (700-ms SOA in Martin et al., 2013 and Foucart et al., 2014; 725 ms in Tanner, Mclaughlin, Herschensohn, \& Osterhout, 2013; $650 \mathrm{~ms}$ in Tokowicz \& MacWhinney, 2005). Therefore, Experiment 2 used a 700-ms SOA to investigate whether non-native speakers would show evidence of form or meaning preactivation when reading more slowly.

\section{Method}

Participants. Participants were 24 Spanish-English bilinguals (10 men and 14 women; age $M=27.4$ years, $S D=4.8$ ), who did not participate in any of the previously described experiments. All participants were tested at University of Edinburgh, and all were native speakers of Spanish. All participants were right-handed and reported no neurological or language disorders. The mean age of their first exposure to English was 10.3 years $(S D=6.2)$, and the mean length of their exposure to English was 15.7 years $(S D=7.7)$. Their overall self-rated English proficiency score was 7.8 ( $S D=1.2$; rated on a scale from 1 to 10$)$. These background measures did not differ from those reported in Experiment $1(p s>1)$.

Stimuli and experimental design. The stimuli and experimental design were identical to those used in Experiment 1.

Procedure and data analysis. The procedure, EEG data processing and statistical analysis were all identical to Experiment 1, except that the SOA was changed to $700 \mathrm{~ms}$ (500-ms word duration, 200-ms interword-interval; sentence final words had an 800-ms duration). Mean accuracy for comprehension questions was $88.9 \%$ ( $S D=11.0 ; 10.4 \%$ of the data was excluded due to time-outs). The mean number of artifact-free trials in Experiment 2 per condition was $38(S D=.23)$, with no difference across conditions.

\section{Results}

As in Experiment 1, the implausible conditions elicited visibly larger N400s than the predictable condition (Figure 2). Notably, unlike Experiment 1, the form condition elicited a larger LPC at posterior channels compared to the other conditions.

The $\mathbf{N 4 0 0}$ time window. The analysis revealed a significant effect of condition, $F(3,69)=6.0, M S E=15.5, p=.001$, and a significant interaction of condition by anteriority, $F(3,69)=3.2$, $M S E=1.0, p<.05$, which stemmed from a stronger effect of condition at posterior channels, $F(3,69)=7.4, M S E=4.4, p<$ .001 , than at anterior channels, $F(3,69)=4.1, M S E=3.9, p=$ .01. Pairwise comparisons between the conditions at posterior channels revealed that all the implausible conditions elicited larger N400s than the predictable condition ( $p$ s $<.001$ ). Critically, the semantic condition elicited a smaller N400 than the unrelated condition, $(M=.82 \mu \mathrm{V}, S D=2.5), t(47)=2.3, p<.05$. There were no differences between any other condition pairs $(p s>1)$.

The post-N400 time window. The analysis revealed a significant effect of condition, $F(3,69)=3.6, M S E=13.6, p<.05$, and a significant interaction of condition by anteriority, $F(3,69)=5.4$, $M S E=1.1, p<.01$, which was driven by the effect of condition being significant at posterior channels only, $F(3,69)=5.1$, $M S E=4.0, p<.01$. Pairwise comparisons at posterior channels revealed that the form condition elicited a larger positive ERP compared to the predictable condition, $(M=2.1 \mu \mathrm{V}, S D=3.0)$, $t(47)=4.8, p<.001$, the semantic condition, $(M=1.7 \mu \mathrm{V}, S D=$ $2.4), t(47)=5.0, p<.001$, and the unrelated condition, $(M=1.4$ $\mu \mathrm{V}, S D=3.0), t(47)=3.3, p<.01$.

Effects of cloze probability. Effects of cloze probability were tested using the same median split approach as in Experiment 1, including posterior channels only. Because there was no N400 reduction for the form condition, we performed a two-way condition (semantic vs. unrelated) by cloze ANOVA in the N400 window, which revealed a marginally significant effect of cloze, $F(1$, 23) $=2.9, M S E=5.4, p=.1$, but no Condition $\times$ Cloze interaction $(F<1)$. In the post-N400 LPC time window, a twoway Condition ( 4 conditions) $\times$ Cloze ANOVA revealed no effect or interaction involving cloze $\left(F_{\mathrm{s}}<1.4\right)$. Following Experiment 1 , we also tested the interaction of condition by cloze in the subset of items (cloze $>30 \%$ ) and in the subset of participants (length of exposure $>4$ years; 21 participants). For the item subset analysis, the ANOVAs in the N400 time window and in the post-N400 time window did not show any significant main effect or interaction involving cloze $(F \mathrm{~s}<1.1)$. For the participant subset analysis, the ANOVAs in the N400 time window and in the post-N400 time window did not show any significant effect or interaction involving cloze either $(F \mathrm{~s}<1)$.

Unlike the results for native speakers, the current LPC effect for non-native speakers seemed independent of the cloze value of the predictable word. However, we noted the possibility that this absence of a cloze-effect was driven by earlier differences (in the 0 - to 200-ms time window) in medium-cloze items. In particular, the form-related condition elicited more positive-going ERPs in this early time window compared to the predictable condition and the unrelated condition. ${ }^{4}$ Such early differences may reflect differences in accidental background fluctuations (i.e., "noise" that is

\footnotetext{
${ }^{4}$ A two-way condition (predictable vs. form) by cloze ANOVA at posterior channels in 0 -ms to 200 -ms time window revealed a significan effect of condition, $F(1,23)=8.3, M S E=8.4, p<.01$, and a significant interaction of condition by cloze, $F(1,23)=4.9, M S E=5.7, p<.05$.
} 
Non-native speakers: All items (700 ms SOA)

"The student is going to the library to borrow a hook/ page/ sofal book tomorrow."

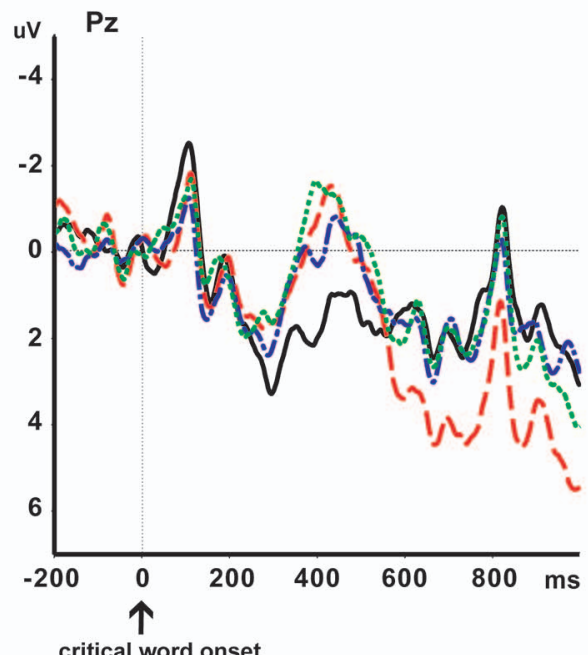

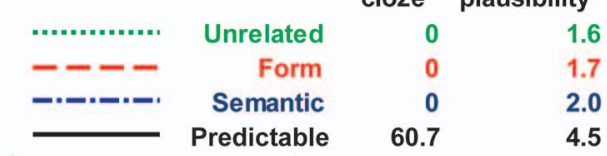
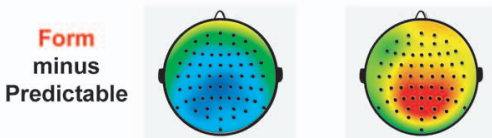

Semantic minus

Predictable

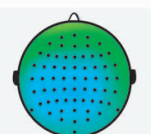

Unrelated minus

Predictable

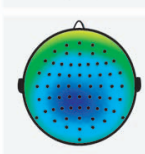

$350-450 \mathrm{~ms}$

$-3 \overline{\mu \mathrm{V} 0 \mu \mathrm{V} 3 \mu \mathrm{V}}-2 \overline{\mu \mathrm{V} 0 \mu \mathrm{V} 2} \mu \mathrm{V}$

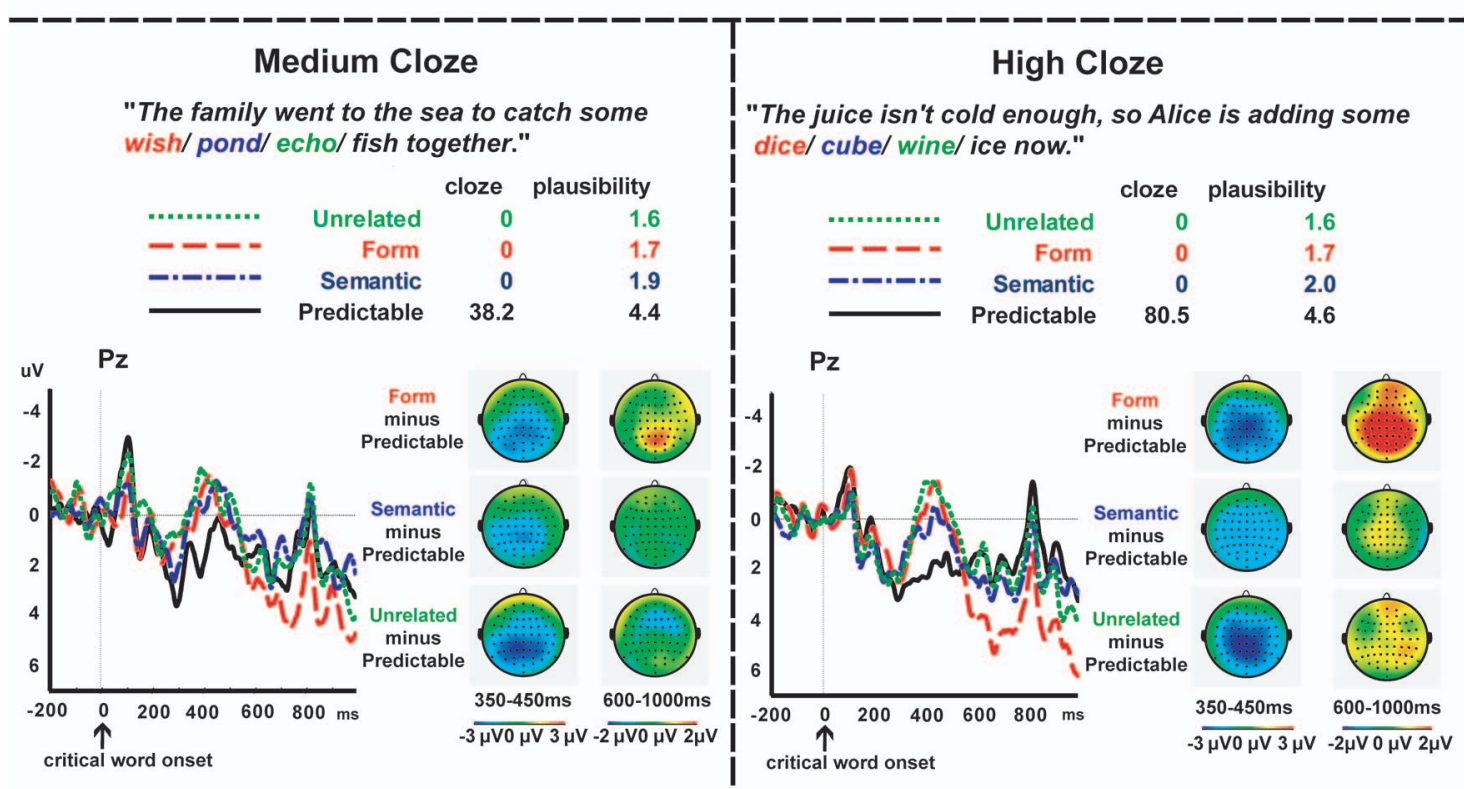

Figure 2. Results from Experiment 2 (700-ms stimulus onset asynchrony [SOA]). Event-related potentials (ERPs) elicited by each condition at $\mathrm{Pz}$ in all items (top panel), in medium-cloze items (left lower panel) and in high-cloze items (right lower panel). Scalp distributions of the ERP effects (implausible condition minus predictable condition) in the N400 time window and LPC (i.e., posterior positivity) time window are shown on the right in each panel. See the online article for the color version of this figure.

not related to the manipulation itself). No such early differences were observed in the high-cloze items. To investigate the effect of cloze without the potential confound of this earlier difference, we reanalyzed our data using a baseline of 0 to $200 \mathrm{~ms}$ after word onset (effectively minimizing the impact of the earlier effect in this window on the later ERP differences). Moreover, in this additional analysis, we used cloze value as a continuous regressor instead of splitting the items into medium-cloze and high-cloze items. Such an analysis provides a more sensitive measure of the graded impact of cloze value.

Additional analyses with cloze value as a continuous regressor. The only change in data preprocessing was the use of a $200 \mathrm{~ms}$ poststimulus time window for baseline correction. For comparability, we plotted the resulting grand-average ERP waveforms in Figure 3. While the overall N400 patterns did not visibly change, it led to a more substantial difference between 
Non-native speakers: All items (700 ms SOA; 0 to $200 \mathrm{~ms}$ baseline)

"The student is going to the library to borrow a hook/ page/ sofal book tomorrow."

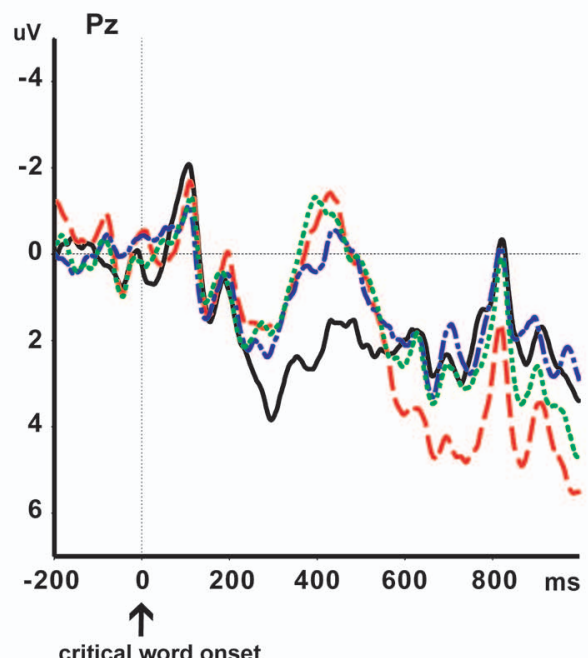

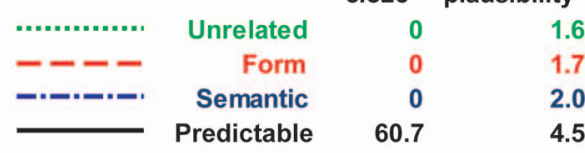
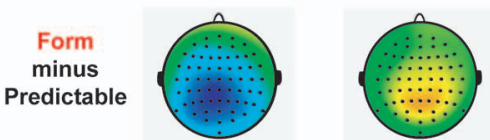

Semantic minus

Predictable

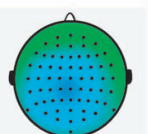

Unrelated minus

Predictable
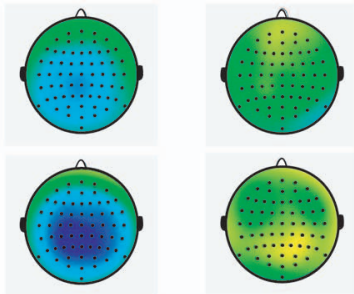

$350-450 \mathrm{~ms}$

$600-1000 \mathrm{~ms}$

$-3 \overline{\mu \mathrm{V} 0 \mu \mathrm{V} 3 \mu \mathrm{V}}-2 \overline{\mu \mathrm{V} 0 \mu \mathrm{V} 2} \mu \mathrm{V}$

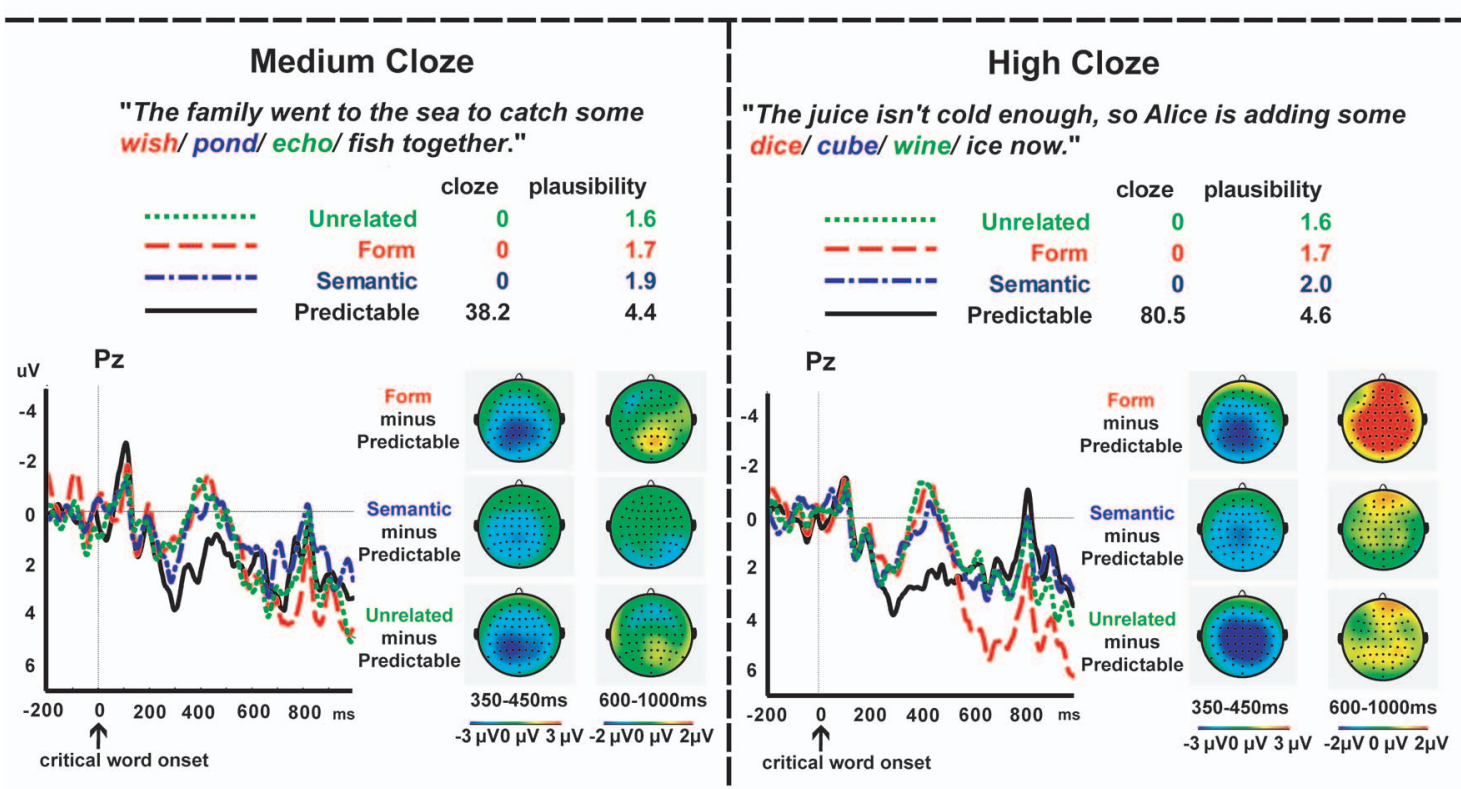

Figure 3. Results from Experiment 2 (700-ms stimulus onset asynchrony [SOA]) with a 200-ms poststimulus baseline. Event-related potentials (ERPs) elicited by each condition at Pz in all items (top panel), in medium-cloze items (left lower panel), and in high-cloze items (right lower panel). Scalp distributions of the ERP effects (implausible condition minus predictable condition) in the N400 time window and LPC (i.e., posterior positivity) time window are shown on the right in each panel. See the online article for the color version of this figure.

the form-related LPC effect in the high-cloze items and in the medium-cloze items. Statistical tests revealed that the overall N400 and LPC effects were not different from our original analysis, and our additional analysis therefore focused only on the impact of cloze value on the LPC effect of form-similarity.

Effects of cloze probability. We tested effects of cloze probability on the LPC effect using linear mixed-effects models, using cloze probability as a continuous predictor. For this analysis, we exported single trial data in the 600- to 1,000 -ms time window at medial-posterior channels (CP1, CP2, CP3, CP4, CPz, P1, P2, P3, $\mathrm{P} 4, \mathrm{Pz})$. We constructed two linear mixed-effects models; the first model evaluated ERP amplitudes as predicted by condition (predictable vs. form), and the second model tested the interaction of condition by cloze in addition to the main effects of condition and 
cloze probability. ${ }^{5}$ The models included random intercept by participant and by item. We assessed the significance of the effects by whether the associated absolute $t$ value exceeded 2 (Baayen, Davidson, \& Bates, 2008). The first model showed a significant effect of condition $(\beta=1.7, S E=.45, t=3.7)$. The second model showed a significant interaction of condition by cloze probability $(\beta=.046, S E=.018, t=2.6) .{ }^{6}$ Effects of condition or cloze were not significant $(t \mathrm{~s}<1.1)$. As shown in Figure 4, higher cloze values were associated with stronger LPC effects.

Between-experiment comparisons: Effects of SOA. We performed a between-experiment comparison to specifically test the impact of SOA on the post-N400 amplitudes in the form condition. We constructed two linear mixed-effects models, one that only used condition (predictable vs. form) as a predictor and a second model that also included an interaction term of condition by SOA. ${ }^{7}$ The first model showed a significant effect of condition $(\beta=-.86, S E=.33, t=-2.6)$, and the second model showed a significant interaction of condition by SOA $(\beta=-1.8, S E=.65$, $t=-2.8$ ). Main effects were not significant in the second model $(t \mathrm{~s}<1)$.

Between-study comparisons: Effects of language nativeness. We also ran linear mixed-effects models to test effects of language nativeness in the N400 (350 to $450 \mathrm{~ms}$ ) and LPC (600 to $1,000 \mathrm{~ms}$ ) time windows. For this analysis, we used high-cloze items in the 700-ms SOA data from native speakers in Ito, Corley et al. (2016) and from non-native speakers in Experiment 2, in which both the N400 reductions and the LPC effect were robust. ${ }^{8}$ The model in the N400 time window evaluated N400 amplitudes as predicted by main effects of condition (predictable vs. unrelated, form vs. unrelated, semantic vs. unrelated) and of language group (native vs. non-native), and the interaction of the two. ${ }^{9}$ The model revealed a significant interaction for the predictable versus unrelated condition comparison $(\beta=-2.6, S E=.98, t=-2.6)$ and for the form versus unrelated condition comparison $(\beta=-2.1, S E=.99$, $t=-2.1)$. These interactions show that the N400 effect for implausible, unrelated words relative to predictable words was greater in native speakers than in non-native speakers, and that the N400 reduction for form-related words relative to unrelated words was found in native speakers but not in non-native speakers. The interaction for the semantic versus unrelated condition comparison was not significant $(t<2)$. This lack of interaction suggests that native- and non-native speakers showed smaller N400s for semantically related words than for unrelated words alike. However, we

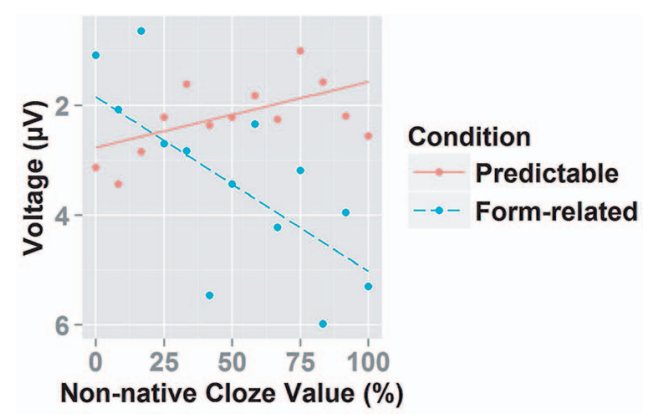

Figure 4. Fitted responses for each of the used cloze values associated with the linear mixed-effects (LME) analysis. See the online article for the color version of this figure. do not interpret the N400 reduction in L2 speakers as clear evidence for prediction of semantic information. This is because, unlike the $\mathrm{N} 400$ reduction in native speakers, the $\mathrm{N} 400$ reduction in non-native speakers did not depend on the cloze values. If the N400 reduction had stemmed from prediction, the degree of the N400 reduction would have been larger in more predictable (higher cloze) sentences than in less predictable (medium-cloze) sentences, as found in native speakers. Given that this was not the case, the N400 reduction likely reflects ease of integration for semantically related words. The plausibility ratings for semantically related words were higher than for unrelated words in highcloze and medium-cloze sets alike, so the integration account can plausibly explain the results. Main effects of condition on all three comparisons and of language group were also significant $(t \mathrm{~s}>2)$. The model in the LPC time window evaluated LPC amplitudes as predicted by main effects of condition (predictable vs. form) and of language group, and the interaction of the two. ${ }^{10}$ The model revealed a significant main effect of condition $(\beta=-2.6, S E=$ $.68, t=-3.7)$. Neither the main effect of language group nor the interaction of condition by language group was significant $(t \mathrm{~s}<$ 1). The result indicates that the LPC effect was similar in nativeand non-native speakers.

\section{Discussion}

In Experiment 2, semantically related words elicited smaller N400s than unrelated words, but this difference did not depend on cloze value. Form-related words elicited a post-N400 positivity at posterior channels (LPC effect). In an additional analysis that corrected for ERP differences in a very early (0 to $200 \mathrm{~ms}$ ) time window, we found that this LPC effect gradually increased in size with increasing cloze probability of the predictable word. Whereas we did not find any evidence for preactivation in non-native speakers in Experiment 2, the LPC results suggest that non-native speakers activated the predictable word after encountering the form-related word, leading to a conflict in interpretation.

\footnotetext{
${ }^{5}$ Model 1: LPC amplitudes condition $+(1 \mid$ participant $)+(1 \mid$ item $)$, Model 2: LPC amplitudes $\sim$ condition ${ }^{*}$ cloze $+(1$ । participant $)+(1$ । item).

${ }^{6}$ An equivalent ANOVA, testing condition (predictable vs. form) by cloze at medial-posterior channels in the 600 -ms to 1,000 -ms time window, revealed a significant interaction of condition by cloze, $F(1,23)=4.9, p<$ .05 , as well as significant effect of condition, $F(1,23)=8.3, p<.01$, but not of cloze, $p>$.1.

${ }^{7}$ Model 1: LPC amplitudes $\sim$ condition $+(1 \mid$ participant $)+(1 \mid$ item $)$, Model 2: LPC amplitudes condition * SOA + (1 I participant $)+(1$ I item), We also ran a model with random slopes for condition by participant and for condition and SOA by item, but this model did not converge.

${ }^{8}$ For the N400 analysis, we used the original $100 \mathrm{~ms}$ prestimulus baseline for both native and non-native speakers. For the LPC analysis, we used the 200-ms poststimulus baseline for the non-native speakers only, following the previous analyses. However, using the original prestimulus baseline for the LPC analysis showed the same pattern of results.

${ }^{9}$ N400 model: N400 amplitudes $\sim$ condition ${ }^{*}$ language group $+(1$ I participant $)+(1$ I item $)$, We also ran a model with random slopes for condition by participant and by item, but this model did not converge.

${ }^{10} \mathrm{~A}$ model with random slopes for condition by participant and by item did not converge.
} 


\section{General Discussion}

We used ERPs to investigate whether non-native speakers preactivate semantic and form information when reading sentences word-by-word at a 500-ms SOA or at a slower 700-ms SOA. These sentences contained a predictable word, an implausible word that was semantically or form-related to that word, or an implausible unrelated word. At both SOAs, predictable words elicited smaller N400s compared with implausible words (i.e., the classic N400 effect). Semantically related words elicited smaller N400s than unrelated words, but unlike the pattern observed in native speakers (Ito, Corley et al., 2016), this N400 reduction did not depend on cloze probability of predictable words. Thus, we did not obtain clear evidence that non-native speakers preactivated form or meaning, although the observed N400 effects do suggest that participants were generally sensitive to sentence plausibility. The second main observation was that form-related words elicited an LPC effect at 700-ms SOA but not at 500-ms SOA. We suggest that this LPC effect reflect the activation of predictable words due to the combined top-down influence of sentence meaning and bottom-up influence of form-similarity. We will discuss the N400 and LPC effects in turn.

\section{No Preactivation of Form or Meaning in Non-Native Speakers}

Our N400 results contrast with previously reported evidence for prediction in non-native speakers (Chambers \& Cooke, 2009; Foucart et al., 2014; Hopp, 2013), adding to a body of evidence suggesting that non-native speaker do not engage in predictive processing as routinely as native speakers do (e.g., Martin et al., 2013).

Because our manipulation did not rely on observing effects on noun-preceding articles that code grammatical gender or the vowel/consonant of the upcoming word, we can rule out an explanation purely based on weaker sensitivity of non-native speakers to grammatical or phonotactic information (e.g., Lew-Williams \& Fernald, 2010). An explanation purely in terms of time constraints does not suffice either, as we did not observe preactivation effects at a relatively slow pace with which a previous ERP study observed preactivation (Foucart et al., 2014).

The specific role of predictability (quantified as cloze probability) and that of proficiency is more elusive, however. Non-native speakers found our predictable words less predictable (average cloze probability of about $60 \%$ ) than native speakers did (average cloze probability of about $80 \%$ ). This is a limitation of the current study, as we did not match predictability between native and non-native speakers. The current effects may signal that the sentences were not constraining enough toward a particular word for non-native speakers, because in our study with native speakers, no preactivation $\mathrm{N} 400$ effects were observed for items with an average cloze probability of about $65 \%$. However, our non-native speakers did not show an N400 prediction effect even in highcloze sentences (average cloze probability of about $80 \%$ ) either. Maybe if we had used extremely predictable sentences (e.g., near$100 \%$ cloze probability in native and non-native speakers), we might have obtained a prediction effect in non-native speakers. ${ }^{11}$ But regardless of whether non-native speakers can in principle predict upcoming information, our results clearly indicate a strong limitation to non-native speakers' likelihood to engage in prediction. Our results fit with the scarce evidence for prediction in non-native speakers; whether prediction occurs can depend on various factors, including the nativeness of the language, as well as cloze probability. All in all, prediction does not appear to be a robust phenomenon, and may not occur so constantly during comprehension, even in native speakers (Huettig \& Mani, 2016; Ito, Martin, \& Nieuwland, 2016).

It remains an open question as to why sentence constraint was lower for non-native speakers than for native speakers. Such constraint differences may arise from different (use of) world knowledge, but could also be a function of language proficiency. An important caveat to explaining our results directly in terms of predictability, however, is that there is no one-to-one correspondence between cloze probability and online prediction effects (e.g., Ito, Corley et al., 2016). Cloze probability is an offline task without time constraint, and therefore gives an estimate of the likelihood that online prediction occurs under generous time constraints (i.e., slow presentation) (Staub, Grant, Astheimer, \& Cohen, 2015). Whereas high-cloze values are associated with higher likelihood of online predictive processing than lower cloze values, high-cloze values are not a guarantee that online prediction occurs. One potential implication of this is that cloze values from nonnative speakers are not as good of an estimation of online prediction as cloze values from native speakers.

Following Kaan (2014), we think that the absence of preactivation effects in our study is primarily due to lower proficiency of non-native speakers. Even when relatively proficient non-native participants read high-constraint sentences at a relatively slow pace, build-up of sentence meaning may not have been fast enough or strong enough to preactivate relevant semantic or form information in advance of the critical words. Compared with native speakers, non-native speakers may then rely more strongly on specific lexical cues to predict upcoming information than on the compositional meaning of the sentence context. Future research should thus tease apart the effects of lexical-associative priming from words in the context and of the exact message conveyed by that context on predictive processing (e.g., Otten \& Van Berkum, 2008).

\section{Activation of Predictable Words Based on Form-Similarity}

At 700-ms SOA, form-related words elicited a larger post-N400 positivity, relative to the predictable condition, which increased in size with higher cloze probability of the predictable word. This cloze-dependent LPC effect was similar to what was found in native speakers at both the 500- and 700-ms SOA (Ito, Corley et

\footnotetext{
${ }^{11}$ To date, evidence for anticipatory processing in non-native speakers has mostly come from visual world eye-tracking (but see Foucart et al., 2014). This may well have to do with sentence constraint, because in those studies the upcoming referents are already visible on the screen, so therefore the sentences have a very high constraint toward a particular continuation (i.e., the equivalent of $100 \%$ cloze probability). In this regard, evidence for predictive/anticipatory processing in visual world paradigms reflects the incremental mapping of incoming language onto given visual information, as opposed to evidence for predictive processing in ERP studies which typically examine brain responses to information associated with words that have not been seen before.
} 
al., 2016) and suggest a conflict in interpretation due to the activation of the predictable word (see also Nieuwland, 2015). The postactivation of predictable words would be stronger in sentences with higher predictability, which will result in greater interpretation conflict. Thus, the LPC effect may be linked to increased difficulty or costs integrating form-related words in high-cloze sentences relative to in medium-cloze sentences. An LPC (P600) effect has been found for sentences that were syntactically difficult to integrate (Kaan, Harris, Gibson, \& Holcomb, 2000). This LPC effect may therefore reflect a certain degree of incremental processing, because it requires a representation of sentence meaning that is sufficiently strong for bottom-up form-similarity to have its effect. This may work through cascaded activation, where the activation of sentence meaning in combination with the formsimilar word leads to activation of the predictable word (see Martin, 2016, for a possible neurocomputational architecture).

Absence of an LPC effect at the 500-ms SOA is consistent with such an interpretation. This SOA is standard for ERP studies on native language comprehension but unusually fast for non-native sentence comprehension (e.g., Foucart et al., 2014; Tanner et al., 2013), and therefore our participants may not have enough time to construct a sufficiently strong or detailed contextual representation. The impact of presentation rate appears to be greater on non-native language comprehension than on native comprehension.

What factors might explain why we did not find evidence of preactivation in non-native speakers, but indeed found LPC evidence for native-like incremental processing? One possibility is that while sentence comprehension is lagging in non-native speakers compared to native speakers, this lag is only short and therefore the representation of sentence meaning in non-native speakers is sufficiently strong by the time that the semantic information associated with the form-related word has been retrieved, giving rise to the conflict in interpretation. This also suggests that incremental processing can, to some extent, proceed normally without prediction via preactivation, or without prediction at all. This is in line with recent views on the role of prediction in language comprehension (e.g., Huettig \& Mani, 2016). Huettig and Mani argued that sentences in everyday conversations are normally less predictive than experimental contexts developed to study prediction. While cloze probability serves a proxy or estimate of processing situations where contextual constraint is particularly high, real language data, and certainly any model of language processing, must span the range of possible cloze values or constraining contexts. If prediction were required for truly incremental language comprehension, then real-world language comprehension could not be the robust, incremental process it is known to be.

\section{Conclusion}

We did not find N400 evidence for preactivation of the form or meaning of upcoming words in non-native speakers. We conclude that although non-native language comprehension can be highly incremental and even predictive (Foucart et al., 2014; Hopp, 2013), non-native speakers may not engage in predictive processing as routinely or robustly as native speakers. However, we observed evidence for the activation of unseen words in an LPC effect to words that were similar in form to a predictable word, which increased gradually with cloze value of the predictable word. We suggest that the combined effects of top-down activation (contextual meaning) and bottom-up activation (form similarity) result in activation of unseen words that fit the context well, thereby leading to a conflict in interpretation reflected in the LPC. This "analysis by synthesis" shows that non-native speakers can use bottom-up and top-down information to constrain incremental interpretation in much the same way that native speakers do.

\section{References}

Baayen, H. R., Davidson, D. J., \& Bates, D. M. (2008). Mixed-effects modeling with crossed random effects for subjects and items. Journal of Memory and Language, 59, 390-412. http://dx.doi.org/10.1016/j.jml .2007.12.005

Bates, E., \& MacWhinney, B. (1989). Functionalism and the competition model. In B. MacWhinney \& E. Bates (Eds.), The crosslinguistic study of sentence processing (pp. 3-76). New York, NY: Cambridge University Press.

Blumenfeld, H. K., \& Marian, V. (2007). Constraints on parallel activation in bilingual spoken language processing: Examining proficiency and lexical status using eye-tracking. Language and Cognitive Processes, 22, 633-660. http://dx.doi.org/10.1080/01690960601000746

Chambers, C. G., \& Cooke, H. (2009). Lexical competition during secondlanguage listening: Sentence context, but not proficiency, constrains interference from the native lexicon. Journal of Experimental Psychology: Learning, Memory, and Cognition, 35, 1029-1040. http://dx.doi .org/10.1037/a0015901

Clahsen, H., \& Felser, C. (2006). Grammatical processing in language learners. Applied Psycholinguistics, 27, 3-42. http://dx.doi.org/10.1017/ S0142716406060024

Dambacher, M., Dimigen, O., Braun, M., Wille, K., Jacobs, A. M., \& Kliegl, R. (2012). Stimulus onset asynchrony and the timeline of word recognition: Event-related potentials during sentence reading. Neuropsychologia, 50, 1852-1870. http://dx.doi.org/10.1016/j.neuropsychologia .2012.04.011

Dussias, P. E., \& Pinar, P. (2010). Effects of reading span and plausibility in the reanalysis of wh-gaps by Chinese-English second language speakers. Second Language Research, 26, 443-472. http://dx.doi.org/10 $.1177 / 0267658310373326$

Federmeier, K. D., \& Kutas, M. (1999). A rose by any other name: Long-term memory structure and sentence processing. Journal of Memory and Language, 41(4), 469-495.

Foucart, A., Martin, C. D., Moreno, E. M., \& Costa, A. (2014). Can bilinguals see it coming? Word anticipation in L2 sentence reading. Journal of Experimental Psychology: Learning, Memory, and Cognition, 40, 1461-1469. http://dx.doi.org/10.1037/a0036756

Hopp, H. (2006). Syntactic features and reanalysis in near-native processing. Second Language Research, 22, 369-397. http://dx.doi.org/10 $.1191 / 0267658306 \mathrm{sr} 272 \mathrm{oa}$

Hopp, H. (2009). The syntax-discourse interface in near-native L2 acquisition: Off-line and on-line performance. Bilingualism: Language and Cognition, 12, 463. http://dx.doi.org/10.1017/S1366728909990253

Hopp, H. (2013). Grammatical gender in adult L2 acquisition: Relations between lexical and syntactic variability. Second Language Research, 29, 33-56. http://dx.doi.org/10.1177/0267658312461803

Huettig, F. (2015). Four central questions about prediction in language processing. Brain Research, 1626, 118-135. http://dx.doi.org/10.1016/ j.brainres.2015.02.014

Huettig, F., \& Mani, N. (2016). Is prediction necessary to understand language? Probably not. Language, Cognition, and Neuroscience, 31, 19-31. http://dx.doi.org/10.1080/23273798.2015.1072223

Ito, A., Corley, M., Pickering, M. J., Martin, A. E., \& Nieuwland, M. S. (2016). Predicting form and meaning: Evidence from brain potentials. 
Journal of Memory and Language, 86, 157-171. http://dx.doi.org/10 $.1016 /$ j.jml.2015.10.007

Ito, A., Martin, A. E., \& Nieuwland, M. S. (2016). How robust are prediction effects in language comprehension? Failure to replicate article-elicited N400 effects. Manuscript submitted for publication.

Ivanova, I., \& Costa, A. (2008). Does bilingualism hamper lexical access in speech production? Acta Psychologica, 127, 277-288. http://dx.doi .org/10.1016/j.actpsy.2007.06.003

Jackson, C. N., \& Bobb, S. C. (2009). The processing and comprehension of wh-questions among second language speakers of German. Applied Psycholinguistics, 30, 603. http://dx.doi.org/10.1017/S014271640999004X

Kaan, E. (2014). Predictive sentence processing in L2 and L1: What is different? Linguistic Approaches to Bilingualism, 4, 257-282. http://dx .doi.org/10.1075/lab.4.2.05kaa

Kaan, E., Harris, A., Gibson, E., \& Holcomb, P. J. (2000). The P600 as an index of syntactic integration difficulty. Language and Cognitive Processes, 15, 159-201. http://dx.doi.org/10.1080/016909600386084

Kotz, S. A. (2009). A critical review of ERP and fMRI evidence on L2 syntactic processing. Brain and Language, 109, 68-74. http://dx.doi .org/10.1016/j.bandl.2008.06.002

Kotz, S. A., \& Elston-Güttler, K. E. (2004). The role of proficiency on processing categorical and associative information in the $\mathrm{L} 2$ as revealed by reaction times and event-related brain potentials. Journal of Neurolinguistics, 17, 215-235. http://dx.doi.org/10.1016/S0911-6044(03)00058-7

Kroll, J. F., \& De Groot, A. M. B. (2005). Handbook of bilingualism: Psycholinguistic approaches. New York, NY: Oxford University Press.

Kroll, J. F., Gullifer, J. W., \& Rossi, E. (2013). The multilingual lexicon: The cognitive and neural basis of lexical comprehension and production in two or more languages. Annual Review of Applied Linguistics, 33, 102-127. http://dx.doi.org/10.1017/S0267190513000111

Kutas, M., \& Hillyard, S. A. (1980). Reading senseless sentences: Brain potentials reflect semantic incongruity. Science, 207, 203-205. http://dx .doi.org/10.1126/science.7350657

Lew-Williams, C., \& Fernald, A. (2010). Real-time processing of gendermarked articles by native and non-native Spanish speakers. Journal of Memory and Language, 63, 447-464. http://dx.doi.org/10.1016/j.jml .2010 .07 .003

Marinis, T., Roberts, L., Felser, C., \& Clahsen, H. (2005). Gaps in second language sentence processing. Studies in Second Language Acquisition, 27, 53-78. http://dx.doi.org/10.1017/S0272263105050035

Martin, A. E. (2016). Language processing as cue integration: Grounding the psychology of language in perception and neurophysiology. Frontiers in Psychology, 7, 120. http://dx.doi.org/10.3389/fpsyg.2016.00120

Martin, A. E., Monahan, P. J., \& Samuel, A. G. (2016). Prediction of agreement and phonetic overlap shape sublexical identification. Language and Speech. [Advance online publication]. http://dx.doi.org/10 $.1177 / 0023830916650714$

Martin, C. D., Thierry, G., Kuipers, J.-R., Boutonnet, B., Foucart, A., \& Costa, A. (2013). Bilinguals reading in their second language do not predict upcoming words as native readers do. Journal of Memory and Language, 69, 574-588. http://dx.doi.org/10.1016/j.jml.2013.08.001

Mitsugi, S., \& MacWhinney, B. (2016). The use of case marking for predictive processing in second language Japanese. Bilingualism: Language and Cognition, 19, 19-35. http://dx.doi.org/10.1017/ S1366728914000881
Nieuwland, M. S. (2014). "Who's he?" Event-related brain potentials and unbound pronouns. Journal of Memory and Language, 76, 1-28. http:// dx.doi.org/10.1016/j.jm1.2014.06.002

Nieuwland, M. S. (2015). The truth before and after: Brain potentials reveal automatic activation of event knowledge during sentence comprehension. Journal of Cognitive Neuroscience, 27, 2215-2228. http:// dx.doi.org/10.1162/jocn_a_00856

Otten, M., \& Van Berkum, J. J. A. (2008). Discourse-based word anticipation during language processing: Prediction or priming? Discourse Processes, 45, 464-496. http://dx.doi.org/10.1080/01638530802356463

Pullum, G. K., \& Zwicky, A. M. (1988). The syntax-phonology interface. In F. J. Newmeyer (Ed.), Linguistics: The Cambridge Survey; Volume I, linguistic theory: Foundations (pp. 255-280). New York, NY: Cambridge University Press.

Roberts, L., \& Felser, C. (2011). Plausibility and recovery from garden paths in second language sentence processing. Applied Psycholinguistics, 32, 299-331. http://dx.doi.org/10.1017/S0142716410000421

Shook, A., \& Marian, V. (2013). The bilingual language interaction network for comprehension of speech. Bilingualism: Language and Cognition, 16, 304-324. http://dx.doi.org/10.1017/S1366728912000466

Staub, A., Grant, M., Astheimer, L., \& Cohen, A. (2015). The influence of cloze probability and item constraint on cloze task response time. Jour nal of Memory and Language, 82, 1-17. http://dx.doi.org/10.1016/j.jml .2015 .02 .004

Tanner, D., McLaughlin, J., Herschensohn, J., \& Osterhout, L. (2013). Individual differences reveal stages of $\mathrm{L} 2$ grammatical acquisition: ERP evidence. Bilingualism: Language and Cognition, 16, 367-382. http:// dx.doi.org/10.1017/S1366728912000302

Tokowicz, N., \& MacWhinney, B. (2005). Implicit and explicit measures of sensitivity to violations in second language grammar: An eventrelated potential investigation. Studies in Second Language Acquisition, 27, 173-204. http://dx.doi.org/10.1017/S0272263105050102

Townsend, D. J., \& Bever, T. G. (2001). Sentence comprehension: The integration of habits and rules. Computational Linguistics, 28, 238-241.

Trenkic, D., Mirković, J., \& Altmann, G. T. M. (2014). Real-time grammar processing by native and non-native speakers: Constructions unique to the second language. Bilingualism: Language and Cognition, 17, 237257. http://dx.doi.org/10.1017/S1366728913000321

Van de Meerendonk, N., Kolk, H. H. J., Chwilla, D. J., \& Vissers, C. T. W. M. (2009). Monitoring in language perception. Linguistics and Language Compass, 3, 1211-1224. http://dx.doi.org/10.1111/j.1749818X.2009.00163.X

Weber-Fox, C. M., \& Neville, H. J. (1996). Maturational constraints on functional specializations for language processing: ERP and behavioral evidence in bilingual speakers. Journal of Cognitive Neuroscience, 8 , 231-256. http://dx.doi.org/10.1162/jocn.1996.8.3.231

Williams, J. N. (2006). Incremental interpretation in second language sentence processing. Bilingualism: Language and Cognition, 9, 71-88. http://dx.doi.org/10.1017/S1366728905002385

Wlotko, E. W., \& Federmeier, K. D. (2015). Time for prediction? The effect of presentation rate on predictive sentence comprehension during word-by-word reading. Cortex, 68, 20-32. http://dx.doi.org/10.1016/j .cortex.2015.03.014 


\section{Appendix}

\section{Experimental Sentences Along With Plausibility Ratings and Cloze Values of Non-Native Participants}

All 160 experimental sentences are listed below with critical words for each of the four conditions (predictable word, formrelated word, semantically related word, unrelated word, respectively). The mean non-native plausibility ratings for the four conditions per sentences are shown in brackets after each critical word. The mean non-native cloze value of the predictable word is shown in brackets after each sentence.

1. The student is going to the library to borrow a book (4.8)/ hook (1.2)/ page (2)/ sofa (1.6) tomorrow. (100)

2. Living alone is too expensive, so the students will share a flat (4.8)/ flag (1.8)/ wall (1.4)/ bell (1.2) together. (67)

3. The family enjoyed the sunny day, but there will be rain (4.4)/ pain (2)/ sky (1.6)/ loss (1.2) tomorrow. (50)

4. Jack studied medicine in a university and works as a doctor (5)/ factor (1.2)/ patient (1.2)/ tenant (2.4) now. (83)

5. Amelia got a driving license, and will buy her own car (5)/ jar (1.6)/ tire (2)/ rat (1.6) soon. (83)

6. Oliver doesn't have a watch, so he doesn't know the time (4.4)/ lime (1)/ rest (2)/ bean (1.2) now. (83)

7. Rachel will go to the cinema to watch a new film (4.6)/ firm (1.2)/ camera (1.4)/ bird (1.8) tomorrow. (50)

8. Paul is trying to stand on one leg (5)/ lag (2)/ hip (2.4)/ kid (2.2) now. (17)

9. The gambler kept losing, so he doesn't have any money (4.8)/ honey (1)/ wallet (1.8)/ candle (1.2) left. (58)

10. Harry intends to propose to Emily and give her the ring (5)/ wing (1.2)/ finger (1.6)/ memo (2) tomorrow. (92)

11. John nervously asked the attractive girl out on a date (4.8)/ gate (2)/ cancel (1)/ pin (1) yesterday. (67)

12. As a lifetime vegetarian, Olivia doesn't miss eating meat (4.4)/ mean (1.4)/ flour (2)/ soil (1.2) now. (83)

13. Dylan got lost today, so he will use a map (4.6)/ cap (2)/ globe (1.8)/ job (1) tomorrow. (58)

14. The comedian was funny, despite a bad joke (4.8)/ coke (1.6)/ laugh (3.8)/ beef (1) yesterday. (75)
15. Jacob found he misspelled the word (4.6)/ lord (1.4)/ usage (3)/ oven (1.8) earlier. (67)

16. Oscar opened the postbox, and found a letter (4.2)/ litter (1.4)/ heading (1.6)/ birth (1) there. (58)

17. After struggling with the question, Jessica got the answer (4.8)/ dancer (1.4)/ inquiry (3.8)/ pension (2.4) finally. (92)

18. At the airport, James checked in for his flight (4.8)/ sight (2)/ rocket (2.4)/ machine (1.4) earlier. (50)

19. The lottery gave Emily a car as a prize (3.8)/ price (4.4)/ medal (2.2)/ child (2.2) yesterday. (67)

20. Sophie couldn't recall the recent event, and blamed her bad memory (4.8)/ melody (2)/ storage (2)/ eraser (1.2) yesterday. (83)

21. The shoes were small, so Lily asked for the largest size (4.8)/ sign (1.2)/ height (2.6)/ flip (1.4) available. (58)

22. To view the $3 \mathrm{D}$ image, people wore special glasses (4.6)/ classes (2.2)/ eyes (2)/ markets (1.8) yesterday. (92)

23. At the football match, Bob scored a goal (4.8)/ coal (1)/ team (1.4)/ bear (1.8) yesterday. (75)

24. Grace put too much dressing on her salad (4.8)/ ballad (1.4)/ refrigerator (2.2)/ movie (1.2) yesterday. (83)

25. Chloe couldn't afford the necklace because of its high price (4.8)/ pride (1.8)/ seller (1.2)/ radio (1.4) sadly. (58)

26. The family went to the sea to catch some fish (4.4)/ wish (1.4)/ pond (2.2)/ echo (1.4) together. (33)

27. Noah missed the final bus, and needed to take a taxi (4.8)/ tax (1.2)/ fare (2.4)/ seed (1.2) yesterday. (42)

28. The workers reported the difficult problem to their boss (4.8)/ bass (1.2)/ job (2.6)/ port (2.4) yesterday. (58)

29. Freya had a serious car accident and is afraid of driving (5)/ thriving (1.2)/ licenses (1.2)/ finding (1.4) now. (17) 
30. The man was convicted for murder and is in prison (5)/ poison (1.4)/ crime (1.4)/ image (1.4) now. (25)

31. Beth loved cooking, and has become a world famous chef (4.4)/ shelf (1.2)/ buffet (1.6)/ aunt (1.4) now. (67)

32. Kyle asked the dentist to pull out the painful tooth (5)/ booth (1.4)/ brush (3)/ grade (1) gently. (75)

33. Having high blood pressure, George reduced his intake of salt (4.2)/ malt (3.8)/ sea (2.8)/ bond (1.6) considerably. (42)

34. Children were excited to see the first snow this winter (4.8)/ printer (1)/ summer (2.8)/ effect (1.4) yesterday. (58)

35. To see the new-born panda, Lucy will go to the zoo (4.8)/ loo (1.4)/ lion (1.2)/ end (1.8) tomorrow. (92)

36. The country girl was overwhelmed to see streets full of people (4.8)/ purple (1.8)/ customs (3.4)/ length (1.6) yesterday. (83)

37. Daisy is nine months pregnant and will have her first baby (4.2)/ bay (3.2)/ nappy (2.8)/ agent (1.8) soon. (50)

38. Students at the train station are rushing to buy a ticket (4.8)/ thicket (1.4)/ platform (1)/ major (1.4) now. (83)

39. Feeling stressed at his workplace, Max relaxed at home (4.6)/ dome (1)/ laundry (3.4)/ beer (1.4) completely. (58)

40. Isabella dyed her hair, but she doesn't like the color (4.2)/ cutter (2.2)/ paint (2)/ grape (2.6) now. (50)

41. For parking illegally, William was charged a fine (3.6)/ line (1.2)/ court (1.8)/ rest (1) yesterday. (67)

42. Jim will go swimming and get a suntan at the beach (4.4)/ peach (1.2)/ coconut (1.2)/ drama (1.4) tomorrow. (75)

43. The house is haunted by ghosts (4.4)/ boasts (1.4)/ halloween (1.6)/ eagles (2.2) now. (67)

44. The juice isn't cold enough, so Alice is adding some ice (4.2)/ dice (2)/ cube (3.8)/ wine (1.8) now. (92)

45. The restaurant is always busy, so Leo will book a table (4.6)/ label (2.8)/ chair (2)/ field (2.4) now. (75)

46. The baby is hungry and needs to drink some milk (5)/ silk (1.4)/ cow (1.6)/ debt (1.4) now. (83)
47. Before sending the letter, Daniel licked a stamp (4.8)/ stump (1.2)/ payment (1.6)/ juice (1.4) quickly. (42)

48. The waiter got a generous tip because of his good service (4.2)/ surface (2)/ complaints (2.2)/ million (1.4) yesterday. (50)

49. For their mother's birthday, the children will hold a party (4.6)/ pasty (1.8)/ guest (1.2)/ scene (2.4) tomorrow. (33)

50. Only one cake is left, so Lilly doesn't have a choice (3.6)/ voice (1.4)/ future (1.6)/ minute (1.4) anyway. (17)

51. The men are watching football and drinking beer in the pub (4.8)/ rub (2.2)/ owner (1.2)/ let (2.2) together. (33)

52. The plane crash was avoided by the experienced pilot (3.4)/ pallet (1.4)/ flight (2.6)/ mail (1.2) yesterday. (92)

53. The bus driver charged Rosie a regular fare (5)/ care (1.2)/ cash (2.2)/ twin (1.2) yesterday. (25)

54. Henry was seriously injured but the doctor saved his life (5)/ knife (1.4)/ death (2.2)/ corn (1.8) successfully. (75)

55. Joseph used a lighter to make a fire (5)/ hire (1.2)/ chimney (1.4)/ statue (1.8) easily. (50)

56. The famous dancer performed on the stage (4.8)/ state (2.4)/ actor (1.4)/ cloud (1.6) yesterday. (67)

57. The girls are going to have cocktails and dance in a club (4.4)/ crab (1.6)/ sport (1.4)/ mist (2.2) together. (25)

58. The supporters wished the team good luck (5)/ duck (3)/ yell (1.4)/ view (2.2) yesterday. (92)

59. The cricket player wants his own glove, ball and bat (2.8)/ rat (2.2)/ hit (3)/ sneeze (2) now. (33)

60. Hannah bought a calendar and hung it on the wall (4.4)/ mall (2)/ floor (2.2)/ fruit (1.4) yesterday. (67)

61. The man with the history of self-harm cut his wrist (3.8)/ list (1.8)/ grip (2.6)/ sky (1) suddenly. (8)

62. Susan felt tears coming as she sliced the onion (5)/ union (2.4)/ flavour (1.4)/ error (1.6) carefully. (50)

63. The woman was hit by a truck when crossing the road (4.2)/ load (2.4)/ cyclist (1.8)/ math (1) yesterday. (25) 
64. Tyler knows many cocktail recipes because he works in a bar (5)/ war (1.4)/ pint (1.4)/ joy (1.8) now. (67)

65. Kate didn't like coffee, so she ordered a cup of tea (5)/ team (1.2)/ mug (1.4)/ myth (1.4) instead. (83)

66. Sam plays guitar in a popular band (4.8)/ sand (1.6)/ music (1.8)/ hero (2) now. (42)

67. As the trousers were loose, Tony tightened up his belt (4.8)/ beat (1.4)/ helmet (1.6)/ tube (1.2) yesterday. (75)

68. Meg will go to the park to walk her dog (4.4)/ fog (1.4)/ tail (1)/ tire (2) tomorrow. (83)

69. The terrorist shot and killed five people using a gun (5)/ gum (1.4)/ grip (1.8)/ rib (2) yesterday. (67)

70. The mole was digging a hole (3)/ pole (1.8)/ drill (2)/ mass (1) yesterday. (92)

71. The horse went outside the course, and didn't win the race (4.8)/ lace (2)/ bike (1.2)/ snap (1.4) understandably. (83)

72. Emma loves a bargain, and everything she bought was on sale (3.8)/ safe (2.2)/ refunds (3.2)/ noon (1.2) actually. (58)

73. The king's throne was taken by his first-born son (4)/ ton (1.2)/ father (1.2)/ fox (1.4) yesterday. (17)

74. The camp leader taught children how to pitch a tent (3.6)/ cent (1.4)/ cave (2)/ flaw (2.8) yesterday. (0)

75. He enjoys hiking in the woods because of the fresh air (3.8)/ hair (2.4)/ dust (2.2)/ ray (2.2) outside. (75)

76. All the colleagues have savings accounts at the same bank (4.4)/ back (1.8)/ receipt (1)/ lake (1.4) somehow. (75)

77. Ben went to the gym to swim in the pool (4)/ tool (1.8)/ sink (1.2)/ lump (1) earlier. (92)

78. Adam keeps different breeds of cows in his farm (5)/ harm (1.2)/ yields (2.2)/ navy (1.6) now. (75)

79. For relaxation, Matilda soaked in a hot bath (2.8)/ path (1.4)/ soap (1.4)/ loaf (2.4) yesterday. (33)

80. Lewis lost his memory because of the damage to his brain (4.8)/ grain (1.2)/ surgeries (2.2)/ nation (1.8) yesterday. (83)

81. After shuffling, the croupier asked the guest to select one card (4.4)/ cart (3.4)/ swap (1.6)/ roll (3.2) only. (75)
82. Nobody knows the time as this room has no clock (4.8)/ clerk (2.2)/ alarm (3.6)/ scarf (1.4) now. (75)

83. Ryan refused to invest to avoid taking a risk (5)/ disc (1.4)/ benefit (1.6)/ door (1.2) yesterday. (58)

84. The client immediately signed the contract because it was a good deal (5)/ meal (2.4)/ trust (3)/ flower (1.4) indeed. (75)

85. By closely examining a painting, one can see all the detail (3.8)/ retail (1.6)/ paragraphs (1.8)/ syrup (1.8) clearly. (0)

86. Toby used to walk to school, but now he takes a bus (5)/ bug (1)/ seat (1.8)/ use (1.2) often. (75)

87. In the class, Bella whispered in her friend's ear (4.2)/ rear (1.6)/ sound (2)/ kin (1.8) quickly. (92)

88. Two dogs injured each other when they had a fight (4.6)/ light (1)/ troop (1.2)/ piano (1) yesterday. (92)

89. To make meringue, she separated the whites from five eggs (4.8)/ pegs (1.8)/ nests (1.8)/ toes (1.6) carefully. (100)

90. To make two groups, the lecturer split the class in half (4.4)/ calf (1.2)/ dozen (2)/ lake (1.6) quickly. (42)

91. Seeing buds on trees heralds the arrival of spring (4.2)/ sprint (1.6)/ winter (3.6)/ power (1.4) surely. (75)

92. The store was so busy that the clerk needed help (4.4)/ heap (2)/ lifeguards (1.8)/ fog (1.2) yesterday. (58)

93. The solution didn't work, and Harley lost all hope (4.8)/ hose (1.2)/ regret (2)/ nails (2) yesterday. (33)

94. To remember to buy everything she wanted, Gracie made a list (5)/ lift (1.4)/ volume (1.8)/ disc (1.4) quickly. (92)

95. The computer pointer doesn't move though Tommy is moving the mouse (4)/ mouth (1.2)/ click (3.4)/ lemon (1.4) now. (92)

96. Katie looks much younger than her actual age (4.2)/ ace (1.4)/ birth (2.4)/ oak (1.2) now. (50)

97. After the meal, Matthew asked a waiter to bring the bill (5)/ pill (2)/ tip (2.2)/ mess (1.4) quickly. (67)

98. Hearing the noise outside the classroom, the lecturer closed the door (4.6)/ donor (1.6)/ knob (2.4)/ loan (1) immediately. (100) 
99. Elizabeth doesn't believe Matt since he has told lies (4.8)/ pies (1.2)/ truth (2.2)/ ways (1.4) before. (100)

100. The thief stole the bike easily as it had no lock (4.6)/ look (2.2)/ door (1.6)/ sum (1.4) yesterday. (42)

101. Anna brings a sandwich and a salad for her lunch (5)/ punch (1.4)/ kitchen (2.2)/ issue (1.6) usually. (67)

102. People saw the first spaceship that landed on the moon (5)/ mood (3.8)/ orbit (3.6)/ heel (1.4) together. (75)

103. Children made a wish when they saw a shooting star (4.4)/ scar (1.8)/ galaxy (2.8)/ devil (2.2) yesterday. (92)

104. Maya got a cold and has a runny nose (3)/ pose (1.8)/ chin (1.8)/ term (1.8) now. (92)

105. The country has no war and people live in peace (4.6)/ peak (1)/ treaty (2.2)/ yacht (1.6) today. (83)

106. To make the garden more green, Amy is growing various plants (4.6)/ planets (1.2)/ sunlight (1.2)/ limits (1.6) now. (67)

107. To prevent spreading her cold, Jane is wearing a mask (4.2)/ task (1.8)/ face (1.6)/ pane (1.8) now. (25)

108. The leaking rain was due to a hole in the roof (4.2)/ root (1.8)/ floor (3.4)/ pain (1.2) yesterday. (58)

109. The fisherman was trying to catch fish in a net (4.2)/ jet (2.4)/ gross (1.6)/ man (1.2) earlier. (42)

110. The plant lacks sunlight because it's placed in the shade (4.8)/ shape (1)/ grass (2.4)/ broom (1.6) now. (42)

111. Sophia cannot find a suitable lotion because of her delicate skin (5)/ spin (1.2)/ sweat (2.4)/ copy (1.2) now. (67)

112. The driver was stopped as he exceeded the specified speed (4.8)/ speech (1.6)/ jet (1.8)/ tablet (1.8) yesterday. (67)

113. Conner went down the stairs and sat on the bottom step (3.8)/ stem (1.8)/ process (1.2)/ jail (2.6) slowly. (33)

114. With a high salary, he needs to pay more tax (5)/ wax (1)/ income (1.4)/ ash (1) accordingly. (92)

115. Tilly's sister did the cleaning today, so tomorrow it's her turn (4.8)/ turf (1.2)/ gear (1.4)/ acid (1.4) naturally. (50)

116. There was a recycling campaign to reduce the amount of waste (5)/ paste (1)/ toilets (1.4)/ relief (1.4) more. (50)
117. Jamie bravely stopped the robbery without feeling any fear (5)/ year (1)/ escape (1.4)/ dirt (1.8) yesterday. (67)

118. The attendees can't miss the meeting without a good excuse (5)/ excise (1.2)/ doubt (1.6)/ style (2) tomorrow. (50)

119. Frank will double-check the notification in case there is any change (4.8)/ range (1.4)/ same (1.4)/ land (1.8) tomorrow. (8)

120. Finishing his study abroad, David will return to his own country (5)/ counter (1.8)/ import (1.8)/ puzzle (1.6) finally. (58)

121. The businessman left his laptop on his desk (4)/ dusk (1.2)/ receptionist (2)/ pine (1.4) yesterday. (42)

122. Rose couldn't eat noodles using chopsticks, so used a fork (4.8)/ fort (1.8)/ cup (2.4)/ peer (1.4) instead. (92)

123. The bird cannot fly because it injured its wing (4.2)/ ring (1)/ glide (1.6)/ frog (1.2) earlier. (92)

124. Eliza worried about her breath, so she took an extra mint (5)/ hint (1.6)/ herb (2.6)/ toll (2.4) yesterday. (25)

125. Changing majors required students to fill out a twentypage long form (3.4)/ norm (3.6)/ example (1.4)/ rival (1.6) usually. (50)

126. Violet left the dirty plates and cups in the sink (4.2)/ link (2)/ towel (1.8)/ army (1.8) today. (42)

127. To increase her hair volume, the woman wears a wig (3.8)/ pig (1)/ comb (2.8)/ pea (1.2) usually. (42)

128. Eleanor covered the old ugly floor with a large rug (4.6)/ rum (1.4)/ tie (1.8)/ ham (1.4) completely. (8)

129. The room with bad ventilation got a ceiling fan (4)/ fat (1.4)/ air (2.6)/ kit (1.8) finally. (25)

130. For a removal, Cameron packed the TV into its original box (4)/ boa (1.8)/ lid (1.8)/ oil (1.2) carefully. (58)

131. Selling drugs is against the law (4.6)/ saw (1.8)/ jury (2)/ fee (1.8) today. (83)

132. The waiter wasn't polite, so he didn't receive a good tip (4.6)/ lip (1.4)/ thumb (1.4)/ van (1.6) yesterday. (100)

133. For Christmas, the children are hanging bells on the tree (4.6)/ treat (1.6)/ squirrel (2.2)/ inch (1.8) happily. (75) 
134. To expand their market, the project team made a rough plan (3)/ plank (2.8)/ future (2.2)/ rifle (1.2) together. (8)

135. Because of the storm, the ocean has big waves (4.8)/ caves (1.4)/ surfers (2.4)/ heads (1.8) now. (75)

136. The sales staff forgot to attach the price tag (3.8)/ tug (2)/ name (1)/ eve (1.6) again. (42)

137. To make a pancake easily, Julia used a pancake mix (3.6)/ fix (2.4)/ digestion (1)/ bid (1.8) yesterday. (33)

138. The new variety show appointed the entertainer as a host (4.2)/ post (2.6)/ meeting (1.2)/ drill (1.2) yesterday. (8)

139. Nigel's son should inherit the estate according to his will (3.6)/ pill (2)/ fact (1.6)/ site (2.4) naturally. (42)

140. It's expected to snow as it will get very cold (4)/ old (1)/ hot (1)/ tall (2.2) tomorrow. (92)

141. The explorer in the desert hopes to ride the camel (4)/ caramel (1.8)/ oasis (3.2)/ user (1.6) tomorrow. (83)

142. For the parade, the king's servants will refurbish the entire castle (3.8)/ cattle (3.6)/ lords (2.4)/ depth (2.2) perfectly. (17)

143. Making traditional Indian curry requires using several types of spice (5)/ space (1.4)/ orient (2.4)/ excess (1.6) together. (8)

144. To finish the cake, Scarlet spread the whipped cream (4.4)/ dream (3.2)/ pizzas (1.4)/ fiber (1.8) generously. (75)

145. Students learned how to convert kilometres to miles (4.6)/ piles (1.2)/ riders (1.4)/ veins (1.8) yesterday.

146. To compress the air, the machine applies high pressure (4.4)/ pleasure (2.6)/ relief (2)/ session (1.4) constantly. (92)

147. Before exchanging money, Rebecca asks the exchange rate (4.4)/ fate (2.4)/ pace (1.4)/ exit (1.8) usually. (67)
148. Lydia cannot eat anymore as she is so full (4.6)/ dull (2.4)/ half (1)/ mild (1.6) now. (50)

149. Dogs have a good sense of smell (4.4)/ shell (1)/ nose (2.2)/ cash (1.4) naturally. (58)

150. Laura will eat the ice cream quickly before it melts (4.2)/ meets (1.8)/ boils (1.6)/ opens (2) down. (83)

151. After the main course, Sara checked the dessert menu (4.6)/ venue (3)/ chef (1.6)/ bond (2.4) excitedly. (58)

152. Andrew was late because his train had a delay (4.4)/ decay (1.4)/ time (1.8)/ tone (1.8) again. (58)

153. The bomb expert pinpointed a switch to make the bomb explode (3.2)/ explore (2)/ pour (2.4)/ stretch (1.8) finally. (67)

154. Immigration exposed Lisa to a different culture (3.6)/ vulture (1.6)/ sociology (3.4)/ ginger (1.4) naturally. (42)

155. The bridge was washed away by the flood (4.6)/ blood (1.8)/ soil (1.8)/ glove (1.4) yesterday. (8)

156. The story was far from logical and didn't make any sense (4.8)/ fence (1.2)/ taste (1.6)/ button (1.6) completely. (83)

157. Having no ink or paper, the office workers couldn't print (4)/ point (2)/ erase (2)/ move (1.6) anything. (67)

158. In the tennis lesson, Lauren hit the ball with her racket (4.4)/ rocket (1.8)/ game (2)/ area (1.6) well. (83)

159. Joe has grown his moustache, but will give it a shave (4.2)/ share (1.4)/ hair (1.2)/ turtle (1.2) tomorrow. (8)

160. It's so itchy that Chris can't help scratching the mosquito bite (4.2)/ bike (1.4)/ chew (3)/ tape (1.6) constantly. (83)
Received April 26, 2016

Revision received June 14, 2016

Accepted June 16, 2016 